\title{
Die subnationalen Verwaltungen als Legitimationsgaranten der Europäischen Union - belegt an der europäischen Kohäsionspolitik
}

\author{
Volkmar Kese und Daniel Zimmermann*
}

Fast seit Beginn ihres Bestehens wird der Europäischen Union ein Defizit an demokratischer Legitimation attestiert. Zu geringe Kompetenzen des Europäischen Parlaments und zu wenig demokratische Mitbestimmungsmöglichkeiten der Bürger sind immer wieder als Vorwürfe gegenüber dem politischen System der Europäischen Union zu hören. Diese Vorwürfe werden von einer gewissen Europa-Müdigkeit der Unionsbürger begleitet sowie durch einen daraus resultierenden Vertrauensverlust befördert. ${ }^{1}$ Eines der Hauptprobleme bei der Frage nach der Legitimation europäischer Politik ist insbesondere das Fehlen einer europäischen Öffentlichkeit. ${ }^{2}$ Durch die vielfältigen Informations- und Veröffentlichungsverpflichtungen wird dabei insbesondere der europäischen Kohäsionspolitik ein erhebliches Potenzial zur Schaffung einer solchen europäischen Öffentlichkeit beigemessen. ${ }^{3}$ Dieses Politikfeld scheint zudem ein hohes Legitimationspotenzial aufzuweisen, da es der Europäischen Union erlaubt, mit den Strukturfondsmitteln für die Bürger direkt sichtbare Politikergebnisse zu produzieren. $^{4}$

Gerade der sich über zahlreiche subnationale Verwaltungsebenen erstreckende Vollzug im Rahmen einer kohäsionspolitischen Förderperiode ist durch ein hohes Maß an Zusammenarbeit zwischen der Europäischen Kommission und den mitgliedstaatlichen Verwaltungen gekennzeichnet. ${ }^{5}$ Dies trifft vor allem auf die gemeinsame Programmplanung zu. So müssen die Staaten zu Beginn einer Förderperiode der Europäischen Kommission operationelle Programme zur Prüfung vorlegen, in denen sie ihre Prioritäten und Ziele für eine För-

* Prof. Dr. jur. Volkmar Kese, Professor für Staats-, Verwaltungs- und Europarecht an der Hochschule für öffentliche Verwaltung und Finanzen Ludwigsburg; Geschäftsführender Leiter des Instituts für Angewandte Forschung; Studiendekan des Master-Studiengangs Public Management für Führungskräfte, Ludwigsburg.

Daniel Zimmermann, M.A., akademischer Mitarbeiter, Institut für Angewandte Forschung, Ludwigsburg; externer Doktorand an der Otto-von-Guericke-Universität Magdeburg mit den Promotionsbetreuern Prof. Dr. Wolfgang Renzsch und Prof. Dr. Volkmar Kese.

1 Hans Hugo Klein: Europäische Integration und demokratische Legitimation, Baden-Baden 2011, S. 19-20.

2 Michael Brüggemann: Europäische Öffentlichkeit durch Öffentlichkeitsarbeit? Die Informationspolitik der Europäischen Kommission, Wiesbaden 2008, S. 23.

3 Utz Schliesky: Souveränität und Legitimität von Herrschaftsgewalt, Tübingen 2004, S. 672. In einem weiteren Politikfeld, der Dienstleistungsfreiheit, müssen die subnationalen Verwaltungen öffentlichkeitswirksame Kommunikationsstrukturen aufbauen. Das ist bisher - wie verschiedene Forschungsprojekte zeigen - in nur sehr rudimentärer und möglicherweise auch nicht richtlinienkonformer Weise geschehen. Vgl. Volkmar Kese/Helmut Hopp/Claudia Schneider/David H. Fenner: Verwaltungsmodernisierung durch Europäisierung? Die Europäische Dienstleistungsrichtlinie als Veränderungskatalysator für eine Verwaltung im Wandel. Forschungsschriftenreihe des Instituts für Angewandte Forschung, Band 1, Ludwigsburg 2012, S. 249.

4 Stefan Kienle: Die EU-Strukturpolitik nach 2006, Hamburg 2009, S. 26.

5 Bettina Schöndorf-Haubold: Gemeinsame Europäische Verwaltung. Die Strukturfonds der EG, in: Eberhard Schmidt-Aßmann/Bettina Schöndorf-Haubold (Hrsg.): Der Europäische Verwaltungsverbund. Formen und Verfahren der Verwaltungszusammenarbeit in der EU, Tübingen 2005, S. 25-56, hier S. 33. 
derperiode darlegen. ${ }^{6}$ In der Durchführung dürfen Mittel aus den europäischen Strukturfonds dann nur für solche Förderprojekte verwendet werden, die sich zielkonform mit den in den operationellen Programmen festgelegten Förderprioritäten decken. Bei der Umsetzung einzelner Förderprojekte und dadurch zunehmender Wahrnehmbarkeit erfolgreicher Politik sind neben gesellschaftlichen und privaten Akteuren auch dezentrale öffentliche Akteure, insbesondere Kommunalverwaltungen, beteiligt und verantwortlich. ${ }^{7}$

Ob folglich die programmatischen Ziele der europäischen Kohäsionspolitik und die von der Europäischen Union intendierten Ergebnisse erreicht werden, hängt entscheidend von der Umsetzung auf der subnationalen Ebene ab. Bekanntlich wird im Verwaltungssystem der Europäischen Union Unionsrecht nur in Ausnahmefällen von europäischen Institutionen direkt vollzogen. ${ }^{8}$ Zum größten Teil verantworten die nationalen und insbesondere die subnationalen Verwaltungen im indirekten Vollzug die effiziente und effektive Implementierung europäischer Vorgaben. Damit leisten sie aber auch einen unerlässlichen Beitrag zur Legitimation europäischer Politik und sollten deshalb in den wissenschaftlichen (Legitimations-)Debatten entsprechend berücksichtigt werden. Die Feststellung, dass Verwaltungen als Bestandteil der Exekutive für die Umsetzung und Durchführung von politischen Vorgaben verantwortlich sind, erscheint banal. Die zu beobachtende Vollzugsverflechtung im europäischen Verwaltungsverbund ${ }^{9}$ führt jedoch zu langen Steuerungsketten zwischen der Politikformulierung auf europäischer Ebene und den Bürgern als Steuerungsadressaten. Diese Steuerungsketten führen zu Effizienz- und Effektivitätsverlusten bei der Implementierung von europäischen Vorgaben. ${ }^{10}$

In diesem Aufsatz wird der Beitrag der subnationalen Verwaltungen zur Legitimation europäischer Politik untersucht. Dazu ist es zunächst notwendig, die Debatte um die Legitimation der Europäischen Union so zu skizzieren, wie sie gegenwärtig in den Rechts- und Politikwissenschaften geführt wird. In diesen Debatten werden anschließend die subnationalen Verwaltungen verortet und als Legitimationsgaranten beschrieben. Dazu ist es wichtig, ihre Rolle bei der Politikimplementierung im europäischen Mehrebenensystem sowohl rechtlich als auch politisch zu kennzeichnen. Eine Exemplifizierung des Beitrags der subnationalen Verwaltungen zur Legitimation europäischer Politik erfolgt schließlich anhand des Leistungsgrades des Verwaltungsmanagements bei der Umsetzung der europäischen Kohäsionspolitik in den deutschen Bundesländern. Auf diese Weise lassen sich sowohl Aussagen über die subnationalen Verwaltungen als Legitimationsgaranten europäischer Politik als auch über die Legitimation der Europäischen Union in ihrer Gesamtheit treffen.

6 Art. 37 Verordnung (EG) Nr. 1083/2006 des Rates vom 11. Juli 2006 mit allgemeinen Bestimmungen über den Europäischen Fonds für regionale Entwicklung, den Europäischen Sozialfonds und den Kohäsionsfonds und zur Aufhebung der Verordnung (EG) Nr. 1260/1999, in: Amtsblatt der EU, Nr. L 210 vom 31. Juli 2006, S. 25-78.

7 Tanja Kopp-Malek: Strukturfondsförderung, in: Hubert Heinelt/Michèle Knodt (Hrsg.): Politikfelder im EUMehrebenensystem. Instrumente und Strategien europäischen Regierens, Baden-Baden 2008, S. 141-156, hier S. 150.

8 Jürgen Schwarze: Europäisches Verwaltungsrecht. Entstehung und Entwicklung im Rahmen der Europäischen Gemeinschaft, Baden-Baden 2005, S. 33.

9 Gabriele Britz: Vom Europäischen Verwaltungsverbund zum Regulierungsverbund? - Europäische Verwaltungsentwicklung am Beispiel der Netzzugangsregulierung bei Telekommunikation, Energie und Bahn -, in: Europarecht 1/2006, S. 46-78, hier S. 50.

10 Ingeborg Tömmel: Das politische System der EU, München 2008, S. 206. 


\section{Wissenschaftliche Debatten zur Legitimation der Europäischen Union}

Die Europäische Union übt Herrschaftsgewalt aus, da sie verbindliche, allgemeingültige politische Entscheidungen mit Relevanz für die nationale und subnationale Ebene trifft. ${ }^{11}$ Legitimität beschreibt den Zustand, in dem diese Herrschaft gerechtfertigt ist. Legitimation bezieht sich hingegen auf den Prozess, der auf die Herstellung von Legitimität abzielt. ${ }^{12}$ Legitimität wird folglich durch Legitimation vermittelt und ist damit ihr Ergebnis. ${ }^{13}$

In den modernen, herrschenden Demokratietheorien wird zwischen zwei Möglichkeiten der demokratischen Legitimation unterschieden, der Input- und Output-Legitimation. In einer repräsentativen Demokratie gelten politische Entscheidungen gemeinhin als legitim, wenn sie den Willen des Volkes widerspiegeln (,government by the people'), was als InputLegitimation bezeichnet wird. ${ }^{14}$ Aus dieser Perspektive kommt es darauf an, herrschaftliche Anforderungen möglichst unverfälscht aus den Präferenzen der Bürger herzuleiten. ${ }^{15}$

Bei der Output-Legitimation hingegen, also der Legitimation über die Ergebnisse politischer Entscheidungsprozesse, sind die Entscheidungen dann als legitim anzusehen, wenn sie auf wirksame Weise das allgemeine Wohl des Gemeinwesens fördern (,government for the people $).{ }^{16}$ Damit Bürger Politik akzeptieren, müssen demnach Ergebnisse erzielt werden. ${ }^{17}$ Auch wenn die alleinige Konzentration auf eine Output-Legitimation als vorrangige Legitimationsquelle die Gefahr in sich birgt, theoretisch völlig ohne demokratische Elemente auskommen zu können, ${ }^{18}$ so besteht ihr wichtiges Potenzial gerade darin, erkannte Mängel auf der Input-Seite in einer repräsentativen Demokratie kompensieren zu können.

\section{Input-Legitimation: Legitimation über die Mitwirkung der Parlamente an politischen Entscheidungen}

Die demokratische Legitimation der Europäischen Union stützt sich auf die organbezogene Konkretisierung des Grundsatzes der repräsentativen Demokratie in Art. 10 Abs. 1 Vertrag über die Europäische Union (EUV). ${ }^{19}$ Aussagen über das Legitimationsniveau der Europäischen Union können anhand der rechtswissenschaftlichen Legitimationskettentheorie getroffen werden, nach der die demokratische Legitimation hoheitlichen Handelns in einer bruchfreien Kette auf die Willensäußerung des Wählers zurückzuführen ist. ${ }^{20}$ Je länger die Legitimationskette, desto größer ist die Gefahr einzuschätzen, dass politische Entscheidungen nur noch bedingt auf den Willen der Bürger zurückzuführen sind.

11 Beate Kohler-Koch/Thomas Conzelmann/Michèle Knodt: Europäische Integration - Europäisches Regieren, Wiesbaden 2004, S. 193.

12 Enrico Peuker: Bürokratie und Demokratie in Europa. Legitimität im Europäischen Verwaltungsverbund, Tübingen 2011, S. 3.

13 Schliesky: Souveränität und Legitimität, 2004, S. $150 \mathrm{ff}$.

14 Fritz W. Scharpf: Regieren in Europa. Effektiv und demokratisch?, Frankfurt am Main/New York 1999, S. 16.

15 Fritz W. Scharpf: Legitimationskonzepte jenseits des Nationalstaats, in: Gunnar Folke Schuppert/Ingolf Pernice/ Ulrich Haltern (Hrsg.): Europawissenschaft, Baden-Baden 2005, S. 705-741, hier S. 708.

16 Scharpf: Regieren in Europa, 1999, S. 20.

17 Arthur Benz: Politikwissenschaftliche Diskurse über demokratisches Regieren im europäischen Mehrebenensystem, in: Hartmut Bauer/Peter M. Huber/Karl-Peter Sommermann (Hrsg.): Demokratie in Europa, Tübingen 2005, S. 253-280, hier S. 267.

18 Manfred G. Schmidt: Demokratietheorien. Eine Einführung, Bonn 2010, S. 402-403.

19 Matthias Ruffert, in: Christian Calliess/Matthias Ruffert (Hrsg.): EUV, AEUV. Das Verfassungsrecht der Europäischen Union mit europäischer Grundrechtecharta. Kommentar, 4. Aufl., Baden-Baden 2011, Art. 10 EUV, Rn. 5.

20 Schmidt: Demokratietheorien, 2010, S. 400. 
Im Falle der Europäischen Union wird in Art. 10 Abs. 2 EUV der Grundsatz der dualen Legitimation aufgegriffen. Die demokratische Rückkopplung der im Rat der Europäischen Union und im Europäischen Rat vertretenen Regierungsmitglieder an die gewählten Parlamente der Mitgliedstaaten ${ }^{21}$ bildet den ersten Legitimationsstrang. ${ }^{22}$ Der zweite Legitimationsstrang für die europäische Ebene ist das Europäische Parlament, das die Unionsbürger unmittelbar repräsentiert. ${ }^{23} \mathrm{Im}$ Vergleich zum ersten Legitimationsstrang liegt hier eine kürzere Legitimationskette vor.

Auch wenn das Europäische Parlament durch den Vertrag von Lissabon einen beachtlichen Kompetenzzuwachs erfahren hat und seitdem im ordentlichen Gesetzgebungsverfahren gemäß Art. 294 Vertrag über die Arbeitsweise der Europäischen Union (AEUV) in 95 Prozent der Politikbereiche gleichberechtigt mit dem Ministerrat über europäische Rechtsakte abstimmt, ${ }^{24}$ vertritt die herrschende Meinung in den Rechtswissenschaften die Auffassung, die auch vom Bundesverfassungsgericht geteilt wird, dass sich die Legitimation der Europäischen Union überwiegend aus den nationalen Parlamenten speist. So konstatiert das Bundesverfassungsgericht schon im Maastricht-Urteil von 1993, dass die Mitgliedstaaten der Europäischen Union als „Herren der Verträge“ weiterhin ausreichend Kontrollrechte über den Fortschritt der europäischen Integration hätten und der Bezugspunkt für die demokratische Legitimation die nationalen Parlamente als Repräsentationsorgane der einzelnen Völker seien. ${ }^{25}$ Im Lissabon-Urteil von 2009 bestätigt das Bundesverfassungsgericht diese Grundhaltung trotz des Kompetenzzuwachses des Europäischen Parlaments, da dieses nicht die Unionsbürger repräsentiere, sondern - aufgrund der mitgliedstaatlichen Kontingentierung der Sitze im Europäischen Parlament - die Völker der Mitgliedstaaten. ${ }^{26}$ Der Vertrag von Lissabon hat die nationalen Parlamente ebenfalls durch die Einführung der sogenannten Subsidiaritätskontrolle gestärkt. ${ }^{27}$ Diese gibt den nationalen Parlamenten ex ante die Möglichkeit einer Subsidiaritätsrüge, wenn das Subsidiaritätsprinzip gemäß Art. 5 EUV im Rahmen des Gesetzgebungsprozesses auf europäischer Ebene offenbar verletzt wird, sowie ex post die Möglichkeit einer Klage vor dem Europäischen Gerichtshof. ${ }^{28}$

Demokratietheoretisch aber kann die Funktionsfähigkeit eines repräsentativen Demokratiemodells auf europäischer Ebene auch nach dem Inkrafttreten des Vertrags von Lissabon aus mehreren Gründen angezweifelt werden. So ist weiterhin der Großteil der Legislativgewalt eindeutig beim Ministerrat und nicht beim Europäischen Parlament vorzufinden. AuBerdem fehlen auf europäischer Ebene nach wie vor die wesentlichen gesellschaftlichen Voraussetzungen für eine funktionsfähige repräsentative Demokratie, wie beispielsweise eine europäische kollektive Identität, ein europäisches Parteiensystem oder eine europäische Öffentlichkeit, ${ }^{29}$ also gerade die materiell-demokratischen Elemente, die die politischen Ergebnisse für den Bürger am deutlichsten wahrnehm- und greifbar machen und so die tra-

21 In zwei Mitgliedstaaten werden die im Europäischen Rat vertretenen Staatschefs zudem direkt von den Bürgern legitimiert.

22 Ruffert, in: Calliess/Ruffert: EUV, AEUV, 2011, Art. 10, Rn. 7.

23 Ebenda, Rn. 6.

24 Elmar Brok: Die neue Macht des Europäischen Parlaments nach ,Lissabon` im Bereich der gemeinsamen Handelspolitik, in: integration 3/2010, S. 209-223, hier S. 219

25 BVerfGE 89, 155 vom 12.10.1993, Rn. 190.

26 BVerfG, 2 BvE 2/08 vom 30.6.2009, Rn. 284.

27 Christoph Henke: Plädoyer für kürzere Legitimationsketten in der Europäischen Union, in: Europarecht 1/2010, S. 118-136, hier S. 135.

28 Christian Calliess, in: Calliess/Ruffert: EUV, AEUV, 2011, Art. 12 EUV, Rn. 10.

29 Gabriele Abels/Annegret Eppler/Jennifer Träsch: Zum „Demokratiedefizit“ der EU - und wie es sich (nicht) abbauen lässt, in: Bürger im Staat 3/2010, S. 256-266, hier S. 259. 
genden Elemente für die Schaffung eines europäisch-demokratischen ,Bürgerbewusstseins` darstellen könnten.

\section{Erhöhung der Input-Legitimation durch partizipative Ansätze?}

Weitere Möglichkeiten zur Erhöhung der demokratischen Legitimation jenseits der repräsentativen Demokratie finden sich in partizipativen Ansätzen, die auf die Herstellung neuer Formen von Öffentlichkeit und Bürgerbeteiligung abzielen. ${ }^{30}$ Mit der Europäischen Bürgerinitiative (EBI) wurde durch den Vertrag von Lissabon hierfür ein Mittel geschaffen. ${ }^{31}$ Nach den Maßgaben des Art. 11 Abs. 4 EUV können Unionsbürger, deren Anzahl mindestens eine Million beträgt und die gemäß Art. 7 Abs. 1 Verordnung über die Bürgerinitiative $^{32}$ aus mindestens einem Viertel der Mitgliedstaaten kommen, eine Bürgerinitiative starten und die Europäische Kommission damit auffordern, Vorschläge zu europäischen Themen zu unterbreiten. Diese ist nach der Prüfung einer EBI jedoch nicht dazu verpflichtet, einer eingebrachten Initiative nachzugehen. ${ }^{33}$

Ob mit dem Partizipationsinstrument der EBI das Entstehen einer europäischen Öffentlichkeit beschleunigt werden kann, ist derzeit noch unklar. ${ }^{34}$ Aussagen hierzu können wahrscheinlich erst nach ersten erfolgreichen Initiativen und den damit einhergehenden Kommunikations- und Debattenformen in den verschiedenen europäischen Teilöffentlichkeiten getroffen werden. Grundsätzlich muss aber konstatiert werden, dass die Input-Legitimation auf europäischer Ebene strukturelle Schwächen aufweist, die nur langfristig im Zuge der allmählichen Entwicklung einer europäischen kollektiven Identität überwunden werden können. ${ }^{35}$

Die EBI wird also in der bisherigen Debatte um Korrekturen eines als zu ,mittelbar' erkannten demokratischen Repräsentativsystems als ein partizipatives Ausgleichsinstrument verstanden. Es soll durch seine Ansätze einer direktdemokratischen politischen Teilhabe des Bürgerwillens ein Korrektiv bei der Politikgenerierung durch die etablierten, formell repräsentativ-demokratischen Institutionen sein. Insofern werden solche plebiszitären Elemente grundsätzlich im Zusammenhang mit der Schwächenkorrektur der Input-Legitimation diskutiert. Andererseits kann es sich aber auch um eine Zwittererscheinung handeln: Denn partizipative Elemente können gerade auch als Verstärker eines materiell-demokratischen Ansatzes im Sinne eines ,people's interests for the people' verstanden werden, um so ,demokratische Ergebnispolitik durch die Bürger für die Bürger' als Ausdruck erhöhter OutputLegitimation zu erzeugen. Deswegen muss nun ein Blickwechsel erfolgen und die Frage gestellt werden, ob durch politische Ergebnisproduzierung für den Bürger politische Legitimation erzeugt werden kann.

\section{Output-Legitimation: Legitimation über die Ergebnisse europäischer Politik}

Das zu beklagende Defizit an demokratischer Input-Legitimation schärft das Bewusstsein dafür, dass das Potenzial der Output-Legitimation für die Europäische Union deutlich größer

30 Abels/Eppler/Träsch: Zum „Demokratiedefizit“ der EU, 2010, S. 260.

31 Ruffert, in: Calliess/Ruffert: EUV, AEUV, 2011, Art. 11, Rn. 14.

32 Verordnung (EU) Nr. 211/2011 des Europäischen Parlaments und des Rates vom 16. Februar 2011 über die Bürgerinitiative, in: Amtsblatt der EU, Nr. L 65 vom 11. März 2011, S. 1-22.

33 Zur EBI siehe auch: Rudolf Hrbek: Die Europäische Bürgerinitiative: Möglichkeiten und Grenzen eines neuen Elements im EU-Entscheidungssystem, in: integration 1/2012, S. 35-50.

34 Abels/Eppler/Träsch: Zum „Demokratiedefizit“ der EU, 2010, S. 260.

35 Michael Gehler: Europa. Ideen, Institutionen, Vereinigung, München 2010, S. 503-504. 
bemessen werden muss. ${ }^{36}$ Hier rücken die Ergebnisse politischer Entscheidungen in den Fokus und somit die materielle Komponente europäischer Politik, ${ }^{37}$ während das institutionelle Zustandekommen im Sinne der Input-Legitimation in den Hintergrund tritt.

Die Output-Legitimation vermag die mangelnde Input-Legitimation auf europäischer Ebene auszugleichen, ${ }^{38}$ jedoch darf die - wie oben schon erwähnt - mangelnde Input-Legitimation auch nicht durch eine Überbewertung der Output-Legitimation überkompensiert werden, da sonst demokratische Grundwerte insgesamt gefährdet würden. Insofern wird in der Politikwissenschaft das Modell einer fragmentierten Demokratie in einem europäischen Mehrebenensystem vorgeschlagen. Im ersten Fragment bleibt die Input-Legitimation überwiegend auf die mitgliedstaatliche Ebene beschränkt. ${ }^{39}$ Im zweiten Fragment gewinnt auf der europäischen Ebene die Output-Legitimation stärker an Gewicht, da hier besser als auf der mitgliedstaatlichen Ebene politische Steuerung für Bürgerinteressen durch effektives Regieren gewährleistet werden kann. ${ }^{40}$ Ein zentraler Faktor für die Output-Legitimation im europäischen Mehrebenensystem ist hier daher die sich an den Messgrößen Effizienz und Effektivität orientierende Leistungsfähigkeit der Politik bei der jeweiligen Problemverarbeitung. ${ }^{41}$

\section{Effizienz und Effektivität als Messgrößen der Output-Legitimation}

Effizienz misst die Relation zwischen eingesetztem Aufwand und angestrebtem Ziel. ${ }^{42}$ Konkret bedeutet Effizienz, dass ein definiertes Ziel entweder mit möglichst geringem Mitteleinsatz oder mit gegebenen Mitteln ein größtmöglicher Erfolg erreicht wird. ${ }^{43}$ Effektivität ist die Messgröße für die Erreichung eines politischen Ziels. ${ }^{44}$ Beide Kriterien sind zentrale Messgrößen für die Output-Legitimation. ${ }^{45}$ Für eine effiziente und effektive Aufgabenerledigung ist es notwendig, dass der Output im Nachhinein bewertbar ist, um festzustellen, inwieweit sich ein gewünschtes Ergebnis (,result') tatsächlich eingestellt hat.

Bei der Output-Legitimation sind außerdem Fragen nach der Problemadäquanz, der Konsensbildungsqualität und der Implementierbarkeit politischer Entscheidungen von Bedeutung. ${ }^{46}$

\section{Die Bedeutung der subnationalen Verwaltungen in den Legitimationsdebatten}

Die Debatte um die demokratische Legitimation der Europäischen Union beschränkt sich aus einer rechtswissenschaftlichen Perspektive überwiegend auf die Input-Legitimation, die

36 Schliesky: Souveränität und Legitimität, 2004, S. 601; siehe auch: Wolfgang Wessels: Die Öffnung des Staates. Modelle und Wirklichkeit grenzüberschreitender Verwaltungspraxis 1960-1995, Opladen 2000, S. 136.

37 Schliesky: Souveränität und Legitimität, 2004, S. 601.

38 Schmidt: Demokratietheorien, 2010, S. 402.

39 Vivian A. Schmidt: Democracy in Europe. The EU and National Polities, Oxford 2006, S. 5.

40 Ebenda, S. 5.

41 Wessels: Die Öffnung des Staates, 2000, S. 136.

42 Eberhard Schmidt-Aßmann: Das allgemeine Verwaltungsrecht als Ordnungsidee. Grundlagen und Aufgaben der verwaltungsrechtlichen Systembildung, Berlin/Heidelberg 2006, S. 381.

43 Eckhard Pache: Verantwortung und Effizienz in der Mehrebenenverwaltung, in: Veröffentlichungen der Vereinigung der Deutschen Staatsrechtslehrer 66. Bundesstaat und Europäische Union zwischen Konflikt und Kooperation, Berlin 2007, S. 106-143, hier S. 115.

44 Schmidt-Aßmann: Das allgemeine Verwaltungsrecht, 2006, S. 381.

45 Peuker: Bürokratie und Demokratie, 2011, S. 193.

46 Martin Nettesheim: Demokratisierung der Europäischen Union und Europäisierung der Demokratietheorie, in: Hartmut Bauer/Peter M. Huber/Karl-Peter Sommermann (Hrsg.): Demokratie in Europa, Tübingen 2005, S. 143-189, hier S. 154. 
sich aus der Beteiligung des Europäischen Parlaments und der nationalen Parlamente speist. Als Legitimationsgaranten werden dementsprechend die nationalen Parlamente gewertet. ${ }^{47}$ Die Exekutive findet keine Beachtung. Das Defizit an Input-Legitimation ist dieser Ansicht nach hauptsächlich auf lange Legitimationsketten zwischen politischen Entscheidungen und den Willensäußerungen der Bürger sowie auf das Fehlen von Grundvoraussetzungen einer repräsentativen Demokratie zurückzuführen. Auch den neueren partizipativen Ansätzen wird keine große Chance eingeräumt, dieses Defizit in absehbarer Zeit zu beheben.

Bei der Output-Legitimation rückt die Bedeutung der Exekutive in den Untersuchungsfokus. Entsprechend dem in den Politikwissenschaften diskutierten Modell der fragmentierten Demokratie vermag die europäische Ebene durch effektives Regieren eine bessere Politiksteuerung in Bezug auf Bürgerinteressen zu gewährleisten als die mitgliedstaatliche Ebene. Problematisch ist hier allerdings, dass Effizienz und Effektivität als die zentralen Messgrößen für die Output-Legitimation durch die komplexe Verflechtung der Ebenen beim Vollzug von Unionsrecht erheblich eingeschränkt werden. ${ }^{48}$ Politikgenerierung und Vollzug fallen in den meisten Fällen auseinander, da der direkte Vollzug von Unionsrecht durch die Organe der Europäischen Union die Ausnahme bleibt. ${ }^{49}$

Die Annahme der Legitimationskettentheorie, dass die demokratische Qualität beeinträchtig wird, je länger die Legitimationskette ist, kann an dieser Stelle für die Output-Legitimation europäischer Politik analog formuliert werden: Je länger die Steuerungsketten zwischen Bürger und Europäischer Union, desto unwahrscheinlicher ist es, dass die gewünschten Ergebnisse erreicht werden können. Durch das Vollzugsgeflecht zwischen Bund, Land und Kommune im föderalen System der Bundesrepublik Deutschland werden die Steuerungsketten zusätzlich verlängert. Die Annahme der fragmentierten Demokratie, dass die Output-Legitimation auf der europäischen Ebene deshalb gewährleistet werden kann, weil anders als auf der mitgliedstaatlichen Ebene besser im Sinne der Bürger durch effektives Regieren politisch gesteuert werden kann, ${ }^{50}$ trifft daher nur dann zu, wenn es gelingt, die nationalen Akteure, insbesondere Länder und Kommunen, auf die Erzielung der intendierten Ergebnisse hin zu orientieren. ${ }^{51}$ Die subnationalen Verwaltungen sind folglich bei näherer Prüfung systemisch im Zusammenhang der Output-Legitimation zu verorten und können diese im europäischen Verwaltungsverbund mitbegründen und sicherstellen beziehungsweise erhöhen.

\section{Die subnationalen Verwaltungen als Garanten der Output-Legitimation im europäischen Verwaltungsverbund}

Die Begrifflichkeit des europäischen Verwaltungsverbundes steht in der derzeitigen rechts- und verwaltungswissenschaftlichen Debatte für das aktuellste Konzept zur Beschreibung der europäischen Verwaltung. ${ }^{52}$ Den Regelungsstand des europäischen Verwaltungsverbundes bildet das Gefüge aus nationalen und europäischen Behörden, Ämtern, Dienststellen, Körperschaften und anderen juristischen Personen, die das Unionsrecht zu vollziehen

47 Cordula Agnes Janowski: Die nationalen Parlamente und ihre Europagremien. Legitimationsgarant der EU?, Baden-Baden 2005, S. 38-46.

48 Benz: Politikwissenschaftliche Diskurse, 2005, S. 268.

49 Schwarze: Europäisches Verwaltungsrecht, 2005, S. 33.

50 Schmidt: Democracy in Europe, 2006, S. 5.

51 Tömmel: Das politische System der EU, 2008, S. 206.

52 Britz: Vom Europäischen Verwaltungsverbund zum Regulierungsverbund, 2006, S. 46. 
haben. ${ }^{53}$ Der Begriff beschreibt den Zustand der europäischen Verwaltungsstrukturen entweder in seiner Gesamtheit oder sektorenspezifisch in Politikfeldern, in denen Verbundelemente besonders stark ausgeprägt sind. ${ }^{54}$

Vollzugsteilung und -verflechtung sind die Grundelemente des europäischen Verwaltungsverbundes. Der Vollzug ist nicht auf einer Verwaltungsebene autark ausgestaltet, sondern die Tätigkeiten der Ebenen sind vertikal und horizontal miteinander verflochten. ${ }^{55}$ Jede Verflechtung im europäischen Verwaltungsverbund trägt zu Ineffizienz, Informations- und Reibungsverlusten bei, die die Effektivität des Unionsrechts schwächen, ${ }^{56}$ was eine Verringerung der Output-Legitimation zur Folge hätte.

\section{Output-Legitimation durch Europafähigkeit}

Die Bürger erwarten von den Verwaltungen der nationalen und subnationalen Ebenen im europäischen Verwaltungsverbund, ,in komplexen Entscheidungssituationen anhand von nachvollziehbaren, vernünftigen Gründen und unter Berücksichtigung einer Vielzahl teils divergierender Interessen aus einer Vielzahl von Handlungsalternativen auszuwählen und die getroffenen Entscheidungen auch umzusetzen“. ${ }^{57}$ Für eine effiziente und effektive Aufgabenerledigung ist dafür ein hohes Maß an Expertenwissen bei den Verwaltungsbediensteten notwendig. ${ }^{58}$

$\mathrm{Zu}$ dem europabezogenen Expertenwissen treten auch noch weitere Kompetenzen, die eine europafähige Verwaltung auszeichnen. Europafähigkeit meint dabei die Gesamtheit von Eigenschaften und Fähigkeiten, die in öffentlichen Verwaltungen idealerweise gegeben sein müssen, um europäische Angelegenheiten wahrnehmen zu können. ${ }^{59}$ Welche Fähigkeiten hier von Bedeutung sind, hängt entscheidend vom Tätigkeitsbereich eines Verwaltungsbediensteten $a b$ und variiert sektorenspezifisch. Am Beispiel des im Folgenden näher zu untersuchenden Politikfelds, der europäischen Kohäsionspolitik, sind auf der Landesebene mehrere Fähigkeiten notwendig. So erfordert die Erstellung der operationellen Programme insbesondere Fähigkeiten zur Strategiebildung unter Einbeziehung des Politikgeschehens auf europäischer und nationaler Ebene sowie Fähigkeiten zur Auswertung sozioökonomischer Daten. Bei der Durchführung der operationellen Programme hingegen sind Fähigkeiten in den Bereichen Evaluierung und Monitoring zur Überwachung der Zielerreichung notwendig sowie Kenntnisse weiterer Managementinstrumente wie zum Beispiel des Controllings, um die Durchführungsphase innerministeriell zielgerichtet zu steuern. Bei der Umsetzung von einzelnen Förderprojekten auf kommunaler Ebene sind Kenntnisse des Projekt-

53 Eberhard Schmidt-Aßmann: Einleitung: Der Europäische Verwaltungsverbund und die Rolle des Europäischen Verwaltungsrechts, in: Eberhard Schmidt-Aßmann/Bettina Schöndorf-Haubold (Hrsg.): Der Europäische Verwaltungsverbund. Formen und Verfahren der Verwaltungszusammenarbeit in der EU, Tübingen 2005, S. 1-23, hier S. 2 .

54 Britz: Vom Europäischen Verwaltungsverbund zum Regulierungsverbund, 2006, S. 47-48.

55 Ebenda, S. 48.

56 Wolfgang Weiß: Der Europäische Verwaltungsverbund. Grundfragen, Kennzeichen, Herausforderungen, Berlin 2010, S. 15.

57 Peuker: Bürokratie und Demokratie, 2011, S. 223.

58 Ebenda.

59 Benedikt Speer: Die Europafähigkeit des öffentlichen Dienstes, in: Siegfried Magiera/Karl-Peter Sommermann (Hrsg.): Europäisierung und Internationalisierung der öffentlichen Verwaltung, Symposium aus Anlass der Emeritierung von Univ. Prof. Dr. Dr. h. c. Heinrich Siedentopf, Speyer 2007, S. 127-148, hier S. 128. 
managements unerlässlich. ${ }^{60}$ Für die effiziente und effektive Implementierung wird die Europafähigkeit der Verwaltung folglich zu einem zentralen Legitimationsfaktor.

\section{Gefährdung der Output-Legitimation einfordernden Europafähigkeit durch das Dogma des Trennungsprinzips?}

Die dualistische Grundstruktur der Verwaltung der Europäischen Union in den direkten und indirekten Vollzug wird vor allem in den (Europa-)Rechtswissenschaften vom Dogma des Trennungsprinzips bestimmt. Nach diesem Prinzip vollziehen die nationalen Verwaltungen europäisches Recht auf der Grundlage des nationalen Rechts. ${ }^{61}$ Die Pflicht der deutschen Länder gegenüber dem Bund als Mitgliedstaat der Europäischen Union zum loyalen Vollzug des Unionsrechts ergibt sich europarechtlich aus der sogenannten gegenseitigen Loyalitätspflicht nach Art. 4 Abs. 3 EUV in Verbindung mit Art. 23 Grundgesetz(GG). Diese Pflicht der Mitgliedstaaten trifft neben den Ländern auch andere nationale Träger öffentlicher Gewalt wie die Kommunen. ${ }^{62}$ Im unmittelbaren Vollzug ist laut herkömmlicher Meinung eine europäische Richtlinie dem Trennungsprinzip zufolge unter der Anwendung der Art. 83-85 GG nach der Umsetzung in nationales Recht durch einen Transformationsakt ausschließlich als nationales Recht zu betrachten. ${ }^{63}$

Verfassungsrechtlich und organisatorisch bleiben die subnationalen Verwaltungen weiterhin Teil der nationalen Exekutiven. Im funktionellen Sinne sind sie aber auch integraler Bestandteil der Exekutivgewalt der Europäischen Union ${ }^{64}$ und werden deshalb von Vertretern der Rechtswissenschaft mittlerweile als „,kodependente Organismen“65 bezeichnet. Hinzu kommt, dass der mitgliedstaatliche Vollzug von Unionsrecht inzwischen in zahlreiche Strukturformen von Kooperationshandeln eingebunden ist. ${ }^{66}$ Nach dem Kooperationsprinzip ist der Vollzug von Unionsrecht durch vielfältige Verfahren der Zusammenarbeit zwischen europäischen und nationalen Verwaltungen gekennzeichnet. ${ }^{67}$ Die Kooperation erfolgt dabei teilweise vertikal zwischen der europäischen und den mitgliedstaatlichen Verwaltungen und teilweise horizontal zwischen den Verwaltungen der Mitgliedstaaten. ${ }^{68}$

Aus diesen Gründen erscheint das Dogma des Trennungsprinzips zwischen direktem und indirektem Vollzug auch in größer werdenden Teilen der Rechtswissenschaft inzwischen nicht mehr zeitgemäß. ${ }^{69}$ Dennoch fördert es hartnäckig auf der subnationalen Verwaltungs-

60 Sabine Schönefeld/Kathrin Mair/Volkmar Kese: Konzept für eine erfolgreiche EU-Öffentlichkeitsarbeit in Kommunen, in: apf, Zeitschrift für die kommunale und staatliche Verwaltung 4/2010, BW 25-30, hier BW 27-30.

61 Schmidt-Aßmann: Das allgemeine Verwaltungsrecht, 2006, S. 381.

62 Carl Otto Lenz, in: Carl Otto Lenz/Klaus-Dieter Borchardt (Hrsg.): EU-Verträge. Kommentar nach dem Vertrag von Lissabon, Köln/Wien 2010, Art. 4 EUV, Rn. 15.

63 Gernot Sydow: Verwaltungskooperation in der EU. Zur horizontalen und vertikalen Zusammenarbeit der europäischen Verwaltungen am Beispiel des Produktzulassungsrechts, Tübingen 2004, S. 76. Diese landläufige Meinung ist jedoch nicht überzeugend: Umgesetztes europäisches Richtlinienrecht scheint zwar institutionellformal ein Produkt des nationalen Legislativorgans zu sein, es ist aber nur Recht in formalem deutschem Gesetzesgewand und wird materiell immer europäisches Recht bleiben.

64 Andrzej Wasilewski: Der Europäische Verwaltungsraum. Bemerkungen über die Europafähigkeit der mitgliedstaatlichen Verwaltungen anhand von Beispielen aus dem Umweltbereich, in: Siegfried Magiera/Karl-Peter Sommermann (Hrsg.): Verwaltungswissenschaft und Verwaltungspraxis in nationaler und transnationaler Perspektive. Festschrift für Heinrich Siedentopf zum 70. Geburtstag, Berlin 2008, S. 131-143, hier S. 131.

65 Schmidt-Aßmann: Das allgemeine Verwaltungsrecht, 2006, S. 384.

66 Sydow: Verwaltungskooperation in der EU, 2004, S. 3.

67 Schwarze: Europäisches Verwaltungsrecht, 2005, S. CIII.

68 Schmidt-Aßmann: Einleitung, 2005, S. 5.

69 Vgl. mit weiteren Nachweisen nur Weiß: Der Europäische Verwaltungsverbund, 2010, S. 13. 
ebene ein solches Amtsverständnis der Bediensteten, die sich in der Regel gerade nicht als funktionelle ,Europa'-Beamte begreifen. Sie begreifen den Vollzug von Unionsrecht als eine Aufgabe unter vielen anderen. ${ }^{70}$ Jedoch erschöpft sich europabezogene Verwaltungstätigkeit eben nicht ausschließlich im Vollzug von Unionsrecht, sondern erfordert eine durch Europawissen und -fähigkeiten entwickelte pro-europäische Grundhaltung ${ }^{71}$ im Amtsverständnis eines Amtsträgers im europäischen Mehrebenensystem.

Denn neben europabezogenem Expertenwissen, -fähigkeiten und -fertigkeiten wird zudem eine, aus der (europa-)verfassungsrechtlichen Loyalitätspflicht abgeleitete, europafreundliche Grundhaltung der Verwaltungsbediensteten zu einem weiteren wichtigen Legitimationsfaktor. Gerade bei der Umsetzung von europäischen Rechtsakten muss auf allen subnationalen Verwaltungsebenen ein (Europa-)Bewusstsein vorhanden sein. Die Messgröße der Effektivität des Vollzugshandelns ,vor Ort' muss sich an den von der europäischen Ebene intendierten (politischen und rechtlichen) Zielen orientieren. Denn dabei handelt es sich nicht um eine vom Amtsträger zu begreifende, individuell-voluntative Aufgabe. Die Verwaltungen und insbesondere ihre Bediensteten müssen dabei begreifen, dass sie als quasi ,Mit-Hüter des europäischen Rechts und Garant europäischer Politikgestaltung' fungieren. Diese Hüter-/Garantenfunktion basiert auf dem alle Staatselemente verpflichtenden objektiven Recht der eigenen nationalen Staatszielbestimmung der Art. 23 und 24 GG in Verbindung mit dem europäischen Loyalitätsprinzip des Art. 4 Abs. 3 EUV.

Nur durch die Wahrnehmung dieser verfassungsrechtlichen Pflicht können die subnationalen Verwaltungen als Legitimationsgaranten fungieren und einen Beitrag zur Kompensation der defizitären Input-Legitimation der Europäischen Union leisten. Eine Bewertung der Funktion als Legitimationsgaranten soll im Folgenden am Beispiel der Verwaltungsnotwendigkeiten zur erfolgreichen Umsetzung europäischer Kohäsionspolitik vorgenommen werden.

\section{Der Garanten-Beitrag der subnationalen Verwaltungen zur Output-Legitimation der europäischen Kohäsionspolitik}

Die europäische Kohäsionspolitik weist ein hohes Legitimationspotenzial auf, da es der Europäischen Union erlaubt, mit den Strukturfondsmitteln sichtbare Ergebnisse für die Bürger zu produzieren und sie aktiv in ihren Lebenslagen zu unterstützen ${ }^{72}$ (Generierung politischer ,results', nicht nur reiner ,outcomes'!). Hier zeigen sich die sich europäisierenden Verwaltungsstrukturen eines vertikalen Verwaltungsverbundes sehr deutlich, indem die Verwaltung der europäischen Kohäsionspolitik bei der Programmplanung sehr stark durch eine Zusammenarbeit zwischen der Europäischen Kommission und den mitgliedstaatlichen Verwaltungen, insbesondere den Landesverwaltungen, gekennzeichnet ist. ${ }^{73}$ Nachdem die durch europäische Verordnungen gesteuerte Erstellung der operationellen Programme durch die subnationalen Verwaltungen von der Europäischen Kommission geprüft wurde, erfolgt die Mittelverwaltung der Strukturfonds durch die zuständigen Landesministerien. Für die Ausschreibung von konkreten Förderprojekten sind die Verwaltungsbehörden der Strukturfonds verantwortlich. Auf diese können sich oftmals auch Kommunen bewerben und damit erstreckt sich der europäische Verwaltungsverbunds-Charakter im europäischen Mehrebe-

70 Sydow: Verwaltungskooperation in der EU, 2004, S. 71.

71 Ebenda, S. 86.

72 Kienle: Die EU-Strukturpolitik, 2009, S. 26.

73 Schöndorf-Haubold: Gemeinsame Europäische Verwaltung, 2005, S. 33. 
nensystem bis auf die letzte staatsorganisatorisch erkennbare Entscheidungseinheit. In der Durchführungsphase der operationellen Programme müssen die zuständigen nationalen Behörden jedoch der Europäischen Kommission regelmäßig über den Fortgang der Strukturfondsinterventionen und die Ergebnisse von Evaluierungen Bericht erstatten.

\section{Die Messgrößen Effizienz und Effektivität in der europäischen Kohäsionspolitik}

In der aktuellen Förderperiode 2007 bis 2013 stehen für die europäische Kohäsionspolitik insgesamt circa 308 Millionen Euro aus den Strukturfonds bereit. Die Strukturfonds bestehen aus dem Europäischen Fonds für regionale Entwicklung (EFRE) und dem Europäischen Sozialfonds (ESF). Mit fast 45 Prozent stellt das Politikfeld in dieser Periode damit inzwischen den größten Teil des Haushalts der Europäischen Union noch vor der Gemeinsamen Agrarpolitik.

Angesichts dieser beachtlichen Finanzmittel ist es nicht verwunderlich, dass es seit einigen Jahren ein Hauptanliegen der Europäischen Union ist, dass die Strukturfondsmittel von den subnationalen Verwaltungen effizient und effektiv verwaltet werden. Um die Effizienz der Strukturfondsinterventionen zu erhöhen, wird eine Reduzierung des Verwaltungsaufwandes und der Bürokratiekosten von der Europäischen Kommission als wirksames Mittel angesehen. ${ }^{74}$ Um den effektiven Mitteleinsatz zu gewährleisten, versucht sie mittels hochwertiger und funktionierender Überwachungs- und Bewertungsverfahren sicherzustellen, dass die Strukturfondsmittel gemäß den kohäsionspolitischen Zielen eingesetzt werden. ${ }^{75}$

Das primärrechtliche Ziel der europäischen Kohäsionspolitik ist nach den Maßgaben von Art. 174 Abs. 2 AEUV die Verringerung der Unterschiede im Entwicklungsstand der Regionen der Europäischen Union. Seit der Förderperiode 2007 bis 2013 dienen die Strukturfonds verstärkt auch dazu, die wachstums- und beschäftigungspolitischen Strategien der Europäischen Union umzusetzen. So lässt sich mit Beginn der aktuellen Förderperiode 2007 bis 2013 eine klare Ausrichtung an den Zielen der Lissabon-Strategie erkennen. Ihr Ziel ist es, die Europäische Union in den wettbewerbsfähigsten und dynamischsten Wirtschaftsraum der Welt zu transformieren. ${ }^{76}$ Die Prioritäten der europäischen Kohäsionspolitik liegen auf der Stärkung von Wachstum, Wettbewerbsfähigkeit und Beschäftigung. ${ }^{77}$ Dafür werden die Regionen, die unter dem Ziel 2 „Regionale Wettbewerbsfähigkeit und Beschäftigung“ förderfähig sind, dazu verpflichtet, einen verbindlichen Prozentsatz von 75 Prozent der Strukturfondsmittel für diese Ziele zu verwenden. ${ }^{78}$ Die Strukturfonds werden damit schon zu strategischen Implementierungswerkzeugen der Lissabon-Strategie. ${ }^{79}$

Dennoch besteht in der aktuellen Förderperiode 2007 bis 2013 noch zu viel Umsetzungsspielraum für die Länder, was zur Folge hat, dass auch Strukturfondsinterventionen getätigt werden können, die keinen gesamteuropäischen Mehrwert aufweisen. ${ }^{80} \mathrm{Im}$ Jahr 2009 schlug

74 Europäische Kommission: Mitteilung der Kommission. Schlussfolgerungen aus dem fünften Bericht über den wirtschaftlichen, sozialen und territorialen Zusammenhalt: Die Zukunft der Kohäsionspolitik, KOM (2010) 642, S. 10.

75 Europäische Kommission: Schlussfolgerungen aus dem fünften Bericht, 2010, S. 6.

76 Europäischer Rat: Schlussfolgerungen des Vorsitzes. Europäischer Rat (Lissabon) 23. und 24. März 2000, SN $100 / 00$, S. 2.

77 Art. 3 Abs. 1 EG-Verordnung Nr. 1083/2006.

78 Art. 9 Abs. 3 EG-Verordnung Nr. 1083/2006.

79 Peter Becker: Die europäische Kohäsionspolitik und ihre Modernisierung, Stiftung Wissenschaft und Politik: SWP-Studie, Berlin 2009, S. 14.

80 Fabrizio Barca: An Agenda for a reformed Cohesion Policy. A place-based approach to meeting European Union challenges and expectations, Brüssel 2009, S. VIII. 
Fabrizio Barca vor, die Effektivität der mitgliedstaatlichen Maßnahmen durch die Konzentration auf wenige Investitionsprioritäten europaweit zu erhöhen. ${ }^{81}$ Diesen Konzentrationsvorschlag aufnehmend wird in der kommenden Förderperiode 2014 bis 2020 daher eine enge Verknüpfung der europäischen Kohäsionspolitik mit den Strategiezielen für ein intelligentes, nachhaltiges und integratives Wachstum, kurz Europa-2020-Strategie, ${ }^{82}$ erfolgen. ${ }^{83}$ Gemäß den Verordnungsentwürfen zur kommenden Förderperiode 2014 bis 2020 sollen die Strukturfondsmittel auf eine geringe Anzahl von Investitionsprioritäten konzentriert werden, die in den Strukturfondsverordnungen vorgegeben werden und eine klare Verbindung zur Europa-2020-Strategie aufweisen. Die Strukturfonds ESF und EFRE sollen auf diese Weise zu wirkungsvollen Umsetzungsinstrumenten der Europa-2020-Strategie werden. ${ }^{84}$ In den jeweiligen nationalen operationellen Programmen müssen nun, in bisher unbekannt stringenter Weise, Umsetzungsstrategien entwickelt und transparent gemacht werden, die genaue Aufschlüsse darüber geben, wie die nationale Politik über die Gestaltung ihrer jeweiligen operationellen Programme nachvollziehbare und vor allem bewertbare Ergebnisse zur Erreichung der Ziele der Europa-2020-Strategie erbringen kann. ${ }^{85}$

Um für die Zielerreichung einen Anreiz zu geben, wird die Mittelvergabe zukünftig ex ante und ex post an Bedingungen, die sogenannten Konditionalitäten, geknüpft. ${ }^{86}$ Diese neustrukturierten Konditionalitäten bestimmen die Rahmenbedingungen für die Auszahlung der Strukturfondsmittel. Sollten die Länder diese Konditionalitäten nicht erfüllen, weil die in den operationellen Programmen festgelegten strategischen Ziele verfehlt werden, kann die Europäische Kommission Sanktionsmechanismen in Form einer Mitteleinbehaltung oder -kürzung verhängen. ${ }^{87}$ Die effiziente und effektive Verwaltung der Strukturfondsmittel wird daher in der kommenden Förderperiode 2014 bis 2020 durch ein neues, viel höheres Maß an Strategieorientierung im Vergleich zur aktuellen Förderperiode weiter an Bedeutung gewinnen.

Im Folgenden werden die Messgrößen der Effizienz und Effektivität im Politikfeld der europäischen Kohäsionspolitik und ihrer Verwaltungsumsetzung etwas näher untersucht. Dabei werden vor allem zentrale Neuerungen der kommenden Förderperiode 2014 bis 2020 gleich mitberücksichtigt, um am Stand der derzeitigen Vollzugsbewertungen der europäischen Kohäsionspolitik vor allem in den Landesverwaltungen möglichen Optimierungs- und Handlungsbedarf bei den zuständigen Behörden auszumachen, damit diese tatsächlich als Legitimationsgaranten europäischer Output-Legitimation gelten können.

81 Ebenda, S. XVII.

82 Europäische Kommission: Mitteilung der Kommission. Europa 2020. Eine Strategie für intelligentes, nachhaltiges und integratives Wachstum, KOM (2010) 2020, S. 26.

83 Art. 4 Nr. 1, Europäische Kommission: Vorschlag für eine Verordnung des Europäischen Parlaments und des Rates mit gemeinsamen Bestimmungen über den Europäischen Fonds für regionale Entwicklung, den Europäischen Sozialfonds, den Kohäsionsfonds, den Europäischen Landwirtschaftsfonds für die Entwicklung des ländlichen Raums und den Europäischen Meeres- und Fischereifonds, für die der Gemeinsame Strategische Rahmen gilt, sowie mit allgemeinen Bestimmungen über den Europäischen Fonds für regionale Entwicklung, den Europäischen Sozialfonds und den Kohäsionsfonds und zur Aufhebung der Verordnung (EG) Nr. 1083/2006, KOM (2011) 615. Der Verordnungsentwurf wird auf europäischer Ebene voraussichtlich Anfang 2013 verabschiedet. Es ist davon auszugehen, dass sich in dem Verordnungsentwurf keine systematischen Veränderungen mehr ergeben werden.

84 Europäische Kommission: Europa 2020, 2010, S. 26.

85 Art. 87 Abs. 2, Europäische Kommission: Vorschlag für eine Verordnung, 2011.

86 Ebenda, S. 10.

87 Art. 18, ebenda. 


\section{Output-Legitimation durch effiziente Umsetzung der europäischen Kohäsionspolitik}

Gemäß der oben genannten Definition ist die Umsetzung der europäischen Kohäsionspolitik dann effizient, wenn eine optimale Verteilung der zur Verfügung stehenden Strukturfondsmittel unter schonendem Mitteleinsatz erfolgt, was einer Präzisierung für die folgende Untersuchung bedarf. Der schonende Mitteleinsatz bezieht sich erstens auf die durch die Umsetzung der europäischen Kohäsionspolitik entstehenden Kosten sowie zweitens auf den schonenden Ressourceneinsatz, insbesondere bezüglich des eingesetzten Personals. Letzterer wird drittens durch eine ressourcenschonende Verwaltungsorganisation begünstigt.

Die Förderlogik der europäischen Kohäsionspolitik beinhaltet das grundsätzliche Problem, dass eine effiziente Mittelverwendung insbesondere in den Ziel-2-Regionen eingeschränkt ist. Dies ist darauf zurückzuführen, dass wirtschaftlich leistungsfähige Mitgliedstaaten einen großen Beitrag zum Haushalt der Europäischen Union leisten, jedoch im Rahmen der europäischen Kohäsionspolitik nur einen relativ kleinen Teil an Strukturfondsmitteln erhalten, von dem wiederum ein großer Anteil für administrative Regelungen und Verpflichtungen verwendet wird. ${ }^{88}$ Diese verursachen Kosten und erfordern einen hohen Personaleinsatz.

In Baden-Württemberg zum Beispiel als Ziel-2-Region fallen circa ein Fünftel der Mittel aus dem EFRE von insgesamt 143 Millionen Euro alleine für Personalkosten an. ${ }^{89}$ Der hohe Personaleinsatz kommt deshalb zustande, weil bei der Verwaltung der Strukturfondsmittel in den Ländern eine Vielzahl von Abteilungen und Referaten unterschiedlicher Fachressorts mitwirken, die oftmals divergierende Interessen verfolgen.

In einer Vielzahl von Ländern sind die Verwaltungsbehörden für die Strukturfonds ESF und EFRE in verschiedenen Fachressorts angesiedelt. Die Verwaltung der EFRE-Mittel fällt häufig in das für wirtschaftliche Themen zuständige Fachressort, wie beispielsweise im Freistaat Bayern oder im Land Brandenburg. In beiden Bundesländern sind die Verwaltungsbehörden für den ESF im Fachressort für Arbeit und Soziales verortet. Ähnliches trifft beispielsweise auch auf die Länder Baden-Württemberg und Nordrhein-Westfalen zu. Jedoch gibt es auch Bundesländer, in denen die Verwaltungsbehörden für den ESF und EFRE demselben Fachressort zugeordnet sind. So sind die Verwaltungsbehörden beider Strukturfonds in den Freistaaten Thüringen und Sachsen jeweils dem Fachressort für Wirtschaft zugeordnet. In Thüringen sind beide Verwaltungsbehörden für ESF und EFRE sogar in einem Referat gebündelt, was jedoch im bundesdeutschen Vergleich die Ausnahme ist.

Dieses Zuständigkeitsgeflecht kann zu Informations- und Reibungsverlusten führen, die sich nachteilig auf die Effizienz der Umsetzung der europäischen Kohäsionspolitik auswirken können. Der Grund dafür liegt darin, dass der Interaktionsraum der Ministerialverwaltung in der Regel das eigene Ministerium ist, was die Kommunikation zwischen den Fachressorts erschwert. ${ }^{90}$ Effizienzverluste entstehen deshalb insbesondere dort, wo die Verwaltungsbehörden in unterschiedlichen Fachressorts verortet sind. Die Kommunikation der Verwaltungsbehörden untereinander ist aber gerade unter Effizienzgesichtspunkten wichtig, da dort oftmals ähnliche Aufgaben, wie beispielsweise die Begleitung einzelner Förderprojekte oder die Berichtspflichten gegenüber der Europäischen Kommission, wahrgenommen werden.

88 Barca: An Agenda for a reformed Cohesion Policy, 2009, S. 57.

89 Südwestpresse: SPD und Grüne rangeln um die Zuständigkeit für Vergabe von EU-Millionen, 15.3.2012.

90 Frank Nullmeier: Strategie und politische Verwaltung. Anmerkungen zum Strategiepotential der Ministerialverwaltung, in: Joachim Raschke/Ralf Tils (Hrsg.): Strategie in der Politikwissenschaft. Konturen eines neuen Forschungsfeldes, Wiesbaden 2010, S. 257-265, hier S. 261. 


\section{Output-Legitimation durch effektive Umsetzung der europäischen Kohäsionspolitik}

Um Aussagen über die Effektivität der Umsetzung der europäischen Kohäsionspolitik durch die subnationalen Verwaltungen treffen zu können, müssen zunächst entsprechende Unterkategorien hierfür gebildet werden. Die Strukturfondsmittel werden erstens dann effektiv verwendet, wenn sie einen Beitrag zu den Zielen der europäischen Kohäsionspolitik, insbesondere den strategischen Zielen der Lissabon-Strategie und zukünftig der Europa-2020-Strategie, leisten. Aussagen über die Effektivität können nur auf der Grundlage von Bewertungsverfahren getroffen werden, die folglich die zweite Unterkategorie bilden. Damit überhaupt eine Legitimation über die Outputs erfolgen kann, müssen die mit den Strukturfondsmitteln erzielten Ergebnisse drittens öffentlichkeitswirksam gegenüber den Bürgern kommuniziert werden.

Nach einer Studie zur Umsetzung der aktuellen Förderperiode 2007 bis 2013 beinhalten die operationellen Programme insbesondere der Ziel-2-Regionen ein klares Vorhaben, wie die Ziele der Lissabon-Strategie umgesetzt werden sollen. ${ }^{91}$ Nach Einschätzung der Europäischen Kommission wird in der Praxis dann aber trotzdem eine Vielzahl der Strukturfondsmittel nach dem Gießkannenprinzip vergeben. ${ }^{92}$ Dieser Vorwurf spricht das Problem an, dass die Strukturfondsmittel mit einer fehlenden Zielgenauigkeit vergeben werden. ${ }^{93}$ Für die Ziel-2-Regionen bedeutet dies, dass die Mittel nicht zu den wachstums- und beschäftigungspolitischen Strategien der Europäischen Union beitragen, sondern auf Grundlage landespolitischer Interessen vergeben werden. Dieser Umstand trägt zu einem ineffektiven Einsatz der Strukturfondsmittel bei.

Hinsichtlich der Bewertung der Ergebnisse nimmt die Europäische Kommission die Funktion einer übergeordneten Kontrollinstanz war, indem sie die Berichte der Mitgliedstaaten über die verpflichtenden Monitoring- und Evaluierungsverfahren auswertet und gegebenenfalls von Sanktionen Gebrauch macht. ${ }^{94}$ In der kommenden Förderperiode 2014 bis 2020 werden die Konditionalitäten zusätzlich als wirksame Sanktionsmittel zur Gewährleistung der Zielerreichung der Strukturfondsinterventionen fungieren. Die Monitoring- und Evaluierungsverfahren sind aber auch für die subnationalen Verwaltungen geeignete Kontrollinstrumente, um die in fondsspezifischen operationellen Programmen formulierte Strategie und Ziele umzusetzen, um damit die Output-Legitimation europäischer Politik sicherzustellen. Die neu zu konzipierenden aufwendigen Verfahren sollten von den zuständigen Behörden weniger als Last, denn als Chance wahrgenommen werden, europäische Strukturfondsmittel im Sinne der Bürger einzusetzen.

Bei den Strukturfondsinterventionen ist es schließlich wichtig, dass die Bürger über die Ergebnisse informiert werden. In einer Förderperiode ist es grundsätzlich die Aufgabe der zuständigen Verwaltungsbehörden, die Bürger über die kofinanzierten Förderprojekte zu informieren, wobei neben der erfolgreichen nationalen Projektbewältigung im gleichen Be-

91 Nordregio: Final Report to the European Commission. Directorate-General for Regional Policy. Evaluation Unit. The Potential for the regional Policy Instruments, 2007-2013, to contribute to the Lisbon and the Göteborg objectives for growth, jobs and sustainable development, Stockholm 2009, S. 109.

92 Walter Deffaa: Rede des Generaldirektors für Regionalpolitik der Europäischen Kommission zur Zukunft der europäischen Strukturfonds am 23.3.2012, abrufbar unter: http://ec.europa.eu/regional_policy/conferences/intelligente spezialisierung 2012/doc/walter_deffaa.pdf (letzter Zugriff: 15.8.2012).

93 Becker: Die europäische Kohäsionspolitik, 2009, S. 13.

94 Kopp-Malek: Strukturfondsförderung, 2008, S. 152. 
deutungsumfang insbesondere der Beitrag der Europäischen Union hervorzuheben ist. ${ }^{95}$ In der neuen Förderperiode 2014 bis 2020 muss sogar jede Verwaltungsbehörde eine Person benennen, die auf Ebene des operationellen Programms für die Kommunikation und Information zuständig ist. ${ }^{96}$ Hier dokumentiert sich eben in der Verwaltungspraxis wieder die oben herausgearbeitete (verfassungs-)rechtlich verpflichtende und damit ihre Garantenstellung auslösende Aufgabe der subnationalen Verwaltungen, die Ergebnisse der Strukturfondsinterventionen gegenüber den Bürgern durch geeignete Publikationsmaßnahmen und eine entsprechende europäische Öffentlichkeitsarbeit möglichst transparent zu kommunizieren.

In den zuständigen Behörden werden die strikten Informations- und Veröffentlichungspflichten jedoch häufig als europäisch induzierter, überbürokratischer Mehraufwand begriffen. Außerdem ,stört' die politisch-administrative Führungsspitze oftmals die durch diese Finanzquellentransparenz bedingte notwendige ,Teilung' des politischen Erfolgs eines Projekts. Dabei ist eine solche transparente Kommunikation erfolgreicher Europapolitik, finanziert durch die Europäische Union, aber durch die subnationalen Verwaltungen erfolgreich politisch und verwaltungsorganisatorisch umgesetzt, ein essenzieller, bisher noch in keiner Weise befriedigend erkannter Bestandteil eines Output legitimierenden Beitrags der subnationalen Verwaltungen zur Legitimation der Europäischen Union.

\section{Lösungsansätze zur Steigerung der Effizienz und Effektivität bei der Umsetzung der europäischen Kohäsionspolitik}

Die subnationalen Verwaltungen können ihrer Aufgabe als Legitimationsgaranten nur dann nachkommen, wenn sie eine effiziente und effektive Implementierung europäischer Politik sicherstellen. Im untersuchten Beispiel der europäischen Kohäsionspolitik fallen durch die administrativen Anforderungen der Europäischen Kommission gerade in den Ziel-2-Regionen in Relation zu den zugewiesenen Strukturfondsmitteln hohe Verwaltungskosten an. Hinzu kommen hohe Personalkosten, da in verschiedenen Ressorts eine Vielzahl von Verwaltungsbediensteten mit der Durchführung der operationellen Programme beschäftigt ist. Aufgrund des interministeriellen Zuständigkeitsgeflechts entstehen lange Steuerungsketten und in der Folge Effizienz- und Reibungsverluste. Diese werden verstärkt, wenn die Verwaltungsbehörden beider Strukturfonds, die ähnliche Aufgaben wahrnehmen, in verschiedenen Fachressorts angesiedelt sind.

Die Effektivität der Strukturfondsinterventionen hängt in entscheidender Weise davon ab, ob sie zu den Zielen der wachstums- und beschäftigungspolitischen Strategien der Europäischen Union beitragen. In der aktuellen Förderperiode 2007 bis 2013 bestehen für die subnationalen Verwaltungen hier noch einige Handlungsspielräume, die aber in der kommenden Förderperiode weiter eingeschränkt werden. Insbesondere die Einhaltung der Konditionalitäten richtet neue Maßstäbe an die Evaluierungs- und Monitoring-Verfahren der zuständigen Verwaltungsbehörden, die für sie zur Überwachung der eigenen Strategie der operationellen Programme dienen. Auch für die Strategiebildung und die Durchführung der Strategie ist es notwendig, dass auf der Landesebene über die Ressortgrenzen hinweg kooperiert wird.

Durch die verfassungsrechtliche Verpflichtung zur Output-Legitimation sollte unter Umständen sogar über eine Umgestaltung der bisherigen Verwaltungspraxis hinsichtlich der

95 Art. 96 EG-Verordnung Nr. 1083/2006; hier kann man sich nicht wie oft üblich mit dem bloßen Hinweis auf europäische Mittelzugabe oder auf kleine Europalogos oder andere Minimalhinweise etwa auf Schriftstelen, Hinweisbannern oder bloße Objektgravuren beschränken.

96 Art. 107, Europäische Kommission: Vorschlag für eine Verordnung, 2011. 
Verwaltung der Strukturfondsmittel mehr in Richtung Zentralisierung und Aufgabenkonzentration nachgedacht werden, um eine effiziente und effektive Umsetzung der europäischen Kohäsionspolitik zu gewährleisten. Dass innerhalb der Landesministerien eine Vielzahl von Ressorts und Abteilungen an der Erstellung und Durchführung der operationellen Programme beteiligt ist, macht eine ressort- und abteilungsübergreifende Zusammenarbeit erforderlich. Eine Methode, die ein interdisziplinäres Zusammenarbeiten ermöglicht, ist das Projektmanagement, ebenso müssen Bewertungsmethoden der SWOT-Analyse ${ }^{97}$ und zieldefinitionsunterstützende Methoden wie die Balanced Scorecard beherrscht werden. ${ }^{98}$ Insbesondere durch die möglichen Sanktionsmechanismen im Anschluss an nicht erfüllte Exante- und Ex-post-Konditionalitäten ist ein auf (Management-)Methoden gestütztes, projektorientiertes Arbeiten auf sämtlichen mit dieser Thematik befassten Ministerial- und Verwaltungsebenen unerlässlich. Es reicht hier eben auch gerade nicht aus, worauf in Experteninterviews auf Ministerialebene immer wieder verwiesen wurde, dass dazu ja die Spezialisten der projektabwickelnden Institutionen zeichneten. Eine ergebnisorientierte und strategieumsetzende Erstellung der operationellen Programme muss nämlich bereits die erkennbaren Probleme der künftigen Projektdurchführung mit in das politisch-strategische Kalkül gezogen haben, um in Zukunft einer verschärften Ex-ante- und Ex-post-Evaluation durch die Europäische Kommission sanktionslos standhalten zu können.

Um die Umsetzung der europäischen Kohäsionspolitik in der kommenden Förderperiode effizienter und effektiver zu gestalten, haben zwar Bundesländer, wie beispielsweise BadenWürttemberg, bereits damit begonnen, In-House-Überlegungen anzustellen und Denkpapiere zu möglichen neuen Organisationsformen zu entwickeln. Diese reichen von ausgelagerten, weisungsgebundenen Agenturmodellen bis hin zu inner-, intra- und interministeriellen Organisationsveränderungen (zum Beispiel in Form eines ressortübergreifenden Steuerungsgremiums). ${ }^{99}$ Der Freistaat Sachsen hat zur Sicherstellung der unionsrechtskonformen Umsetzung der kommenden kohäsionspolitischen Förderperiode 2014 bis 2020 sogar eine Begleitstudie in Auftrag gegeben. ${ }^{100}$ Außerdem bildet die neue Förderperiode einen Themenschwerpunkt des Vorsitzlandes, Brandenburg, in der Europaministerkonferenz von 2012 bis 2013. Zusätzlich sollte hier - unter Hintanstellung von länderspezifischen Prestigegründen - intensiv über eine Ländergrenzen übergreifende Best-Practice-Forschung nachgedacht werden.

\section{Die Garantenfunktion erfordert die Aus- und Weiterbildung von Europafähigkeiten in den subnationalen Verwaltungen}

Auch noch nach Inkrafttreten des Vertrags von Lissabon weist die Europäische Union ein Defizit an demokratischer Legitimation auf. Während sich überwiegend rechtswissenschaftliche Vorschläge zur Erhöhung der demokratischen Legitimation auf die Input-Seite von

97 Die Abkürzung SWOT steht für strengths, weaknesses, opportunities und threats.

98 Volkmar Kese/David Fenner: Überlegungen zur Auswahl der Investitionsprioritäten in der Förderperiode 2014-2020 der europäischen Kohäsionspolitik, Kompetenzzentrum Change-Prozesse im öffentlichen Sektor durch Europäisierung: Discussion Paper 3/2012, S. 4-6.

99 Volkmar Kese/Daniel Zimmermann/Matthias Legner/David Fenner: Überlegungen zu neuen Organisationsformen zur EU-rechtskonformen und strategieerfüllenden Umsetzung der Förderperiode 2014-2020 der europäischen Kohäsionspolitik, Kompetenzzentrum Change-Prozesse im öffentlichen Sektor durch Europäisierung: Discussion Paper 7/2012, S. 5-12.

100 Sächsisches Staatsministerium für Wirtschaft, Arbeit und Verkehr: Auftragsbekanntmachung 2012/S 75-123472, Ex-Ante-Evaluierung für den Europäischen Sozialfonds und den Europäischen Fonds für Regionale Entwicklung im Freistaat Sachsen, in: Amtsblatt der EU, Nr. S 75 vom 18. April 2012. 
Politik beziehen, hebt dieser Aufsatz den Beitrag der subnationalen Verwaltungen als Garanten für die Output-Legitimation europäischer Politik hervor. Die Messgrößen Effizienz und Effektivität sind dabei die zentralen Kriterien für die Output-Legitimation, was am Politikfeld der europäischen Kohäsionspolitik und ihrer Umsetzungsnotwendigkeiten exemplifiziert wurde.

Effizienz- und Effektivitätsverluste entstehen in diesem Politikfeld insbesondere durch lange Steuerungsketten aufgrund eines Zuständigkeitsgeflechts von unterschiedlichen Fachressorts. Hinzu kommt eine häufig ineffektive Mittelvergabe der Strukturfonds.

Die notwendige erhebliche Steigerung der Effizienz und Effektivität bei der Umsetzung der europäischen Kohäsionspolitik in der Förderperiode 2014 bis 2020 zwingt angesichts ihrer herausfordernden Neuerungen zu einer ressort- und abteilungsübergreifenden, vor allem methodisch ausgerichteten Zusammenarbeit in und zwischen den Landesministerien. Hierzu müssen in verstärktem Maße neuartige organisatorische und methodische Managementinstrumente des New Public Managements eingesetzt werden, wie sie in nichtministeriellen Verwaltungsstrukturen schon erfolgreich zur Effizienz- und Effektivitätserhöhung beitragen konnten.

Hinzu kommen weitere Kenntnisse und Fähigkeiten, die mit der Erstellung der operationellen Programme und deren Durchführung verbunden sind, wie einerseits die Ausbildung der Fähigkeiten zur Strategiebildung, andererseits Managementmethoden zur Steuerung und Überwachung der strategischen Zielerreichung.

Die dann so produzierten Ergebnisse wiederum können nur mit eindeutigen Haltungsänderungen des Führungspersonals erzeugt und müssen mit adäquaten Methoden öffentlichkeitswirksam, Output legitimierend politisch ,vermarktet‘ werden.

Dies zeigt deutlich, dass für die Output-Legitimation europäischer Politik, deren Garanten die subnationalen Verwaltungen sind, zumindest sektorenspezifisch neue Europafähigkeiten der Verwaltungsbediensteten unerlässlich sind. Hier lassen sich Entwicklungen auch in anderen europäischen Politikbereichen beobachten, wie zum Beispiel die Vertiefung der Dienstleistungsfreiheit durch die verwaltungsstrukturverändernde Europäische Dienstleistungsrichtlinie, ${ }^{101}$ die immer wieder den Gedanken aufkommen lassen, dass sich eine EUinduzierte Verwaltungsmodernisierung der Mitgliedstaaten in der nächsten Dekade über ein - bewusstes oder (noch) unbewusstes - Implementieren von Instrumenten eines New European Public Managements vollziehen wird. Eine solche Entwicklung gilt es genauestens zu beobachten, denn damit könnte eine Revitalisierung der in den letzten Jahren an Dynamik einbüßenden deutschen Debatte um das New Public Management ausgelöst werden.

101 Richtlinie 2006/123/EG des Europäischen Parlaments und des Rates vom 12. Dezember 2006 über Dienstleistungen im Binnenmarkt, in: Amtsblatt der EU, Nr. L 376 vom 27. Dezember 2006, S. 36-68. 